\title{
Excitation of the 5-day Wave
}

\author{
By Hyeong-Bin Cheong, ${ }^{*)}$ and Ryuji Kimura**) \\ (Communicated by Yoshibumi Tomoda, M. J. A., April 12, 1995)
}

\begin{abstract}
The 5-day wave was detected in composite maps of geopotential height fields of ECMWF data set of 8 years from 1984 to 1991. The structure was similar to that predicted by the Laplace's tidal equation except a characteristic NW-SE meridional phase tilt in the Southern Hemisphere. To understand the cause of this meridional phase tilt, numerical simulations were carried out with the shallow water equation on a sphere (Laplace's tidal equation) with a periodic forcing in a form of a standing wave of zonal wavenumber 1 . The result showed that the meridional phase tilt of the 5 -day wave was produced only when the forcing was located in high latitudes. Comparison of this result with the observational results indicates that a forcing of the 5-day wave is located in the high latitude of the Southern Hemisphere. The standing wave is produced by coupling of the Antarctica with the fluctuating Westerly. Numerical simulations which incorporated this mechanism reproduced the realistic meridional phase tilt of the observed 5-day wave, suggesting that this coupling is an effective mechanism which excites the 5-day wave by resonance.
\end{abstract}

Key words : Free oscillation; atmosphere; Rossby wave; 5-day wave; Antarctica.

Introduction. The 5-day wave is a typical mode of the free oscillation of the global atmosphere, i.e., the gravest, symmetric, external, normal mode Rossby wave with zonal wavenumber 1 . The horizontal structure of the 5-day wave is expressed well by the 1st symmetric Hough modes which are the eigenfunctions of the Laplace's tidal equation with the equivalent depth of $10 \mathrm{~km} .{ }^{1)}$ Since the discovery of the 5-day waves by Madden and Julian (1972), ${ }^{2)}$ a considerable studies were performed on this wave: It was observed not only in the troposphere, ${ }^{3)-8)}$ but also in the stratosphere and higher levels. ${ }^{4), 9), 10)}$ Among these observational analyses, Madden and Julian $(1972)^{2)}$ and Hirota and Hirooka $(1984)^{10)}$ visualized clearly the global structure of the 5-day wave in the troposphere and in the stratosphere, respectively. Though the 5-day wave is observed throughout the year, the amplitude is generally larger in summer of Northern Hemisphere (NH) than in winter of $\mathrm{NH}^{4), 5)}$ It is also known that the presence of the realistic zonal flow does not affect the basic structure of the 5-day wave. ${ }^{11)-13)}$ The similar result was obtained in the barotropic

*) Korian Inter-University Institute of Ocean Science, Pusan Fisheries University, Dae-Yeon Dong Nam-Gu Pusan, Korea. **) Ocean Research Institute, University of Tokyo, 1-15-1 Minamidai, Nakano, Tokyo, Japan. atmosphere. ${ }^{14)}$ However, the forcing mechanism is not known yet in spite of the large amount of observational facts. Although probable forcing mechanisms were suggested by Geisler and Dickinson (1976), ${ }^{11)}$ Salby $(1984),{ }^{15)}$ Salby and Garcia (1987), ${ }^{16)}$ Garcia and Salby $(1987),{ }^{17)}$ there is little observational evidence which shows the form of the forcing. Salby and Garcia $(1987)^{16)}$ showed that the 5-day wave excited by the random forcing localized in the equatorial region had a considerable vertical phase tilt which was not consistent to the observed features.

In this study we try to detect the 5-day wave in a statistical way with data of long periods to see the global structure in the troposphere where the forcing mechanism must be located. Then, we look for a possible forcing mechanism which explains the observed features of the 5-day wave with a numerical model.

Data analysis. We used the objectively analyzed ECMWF (European Centre for Medium-range Weather Forecast) data. The horizontal resolution is 2.5 degrees in both longitude and latitude, and two maps are provided each day. We analyzed the geopotential height fields of 700, 500, 300, 100 and $10 \mathrm{hPa}$ levels. The time series data are subdivided into two seasons, $\mathrm{NH}$-summer and $\mathrm{NH}$-winter: $\mathrm{NH}$-summer is defined 
as six months from April 16 to October 15 and $\mathrm{NH}$-winter is from October 16 to April 15 of the following year. We actually analyzed 7 summers and 7 winters with data processed by a digital band-pass filter of 61 points with cutoff periods of 2.0 and 8.0 days designed according to Hamming (1977). ${ }^{18)}$

The 5-day wave is invisible in an instantaneous weather chart masked by noises due to fluctuations of the atmospheric circulation. However, it can be detected in a correlation map. We used the zonally averaged one-point correlation method ${ }^{19)}$ to detect the 5 -day wave in the preliminary analysis. Fig. 1(a) shows the zonally averaged, one-point correlation map at $500 \mathrm{hPa}$ level in the $\mathrm{NH}$ summer. We followed Randel $(1988)^{19)}$ in the zonally averaging procedure, i.e., a solid dot in the figure represents the reference point on which 18 correlation maps each of which has a reference point located in the different positions on the equator are composited. Therefore, this figure does not contain information of inhomogeneous features in the longitudinal direction. The one-point correlation map clearly indicates that a disturbance with the zonal wavenumber 1 is predominant in the low latitude. The structure is global and nearly symmetric with respect to the Equator. The disturbance propagates westward with a period of nearly 4.5 days, suggesting character-
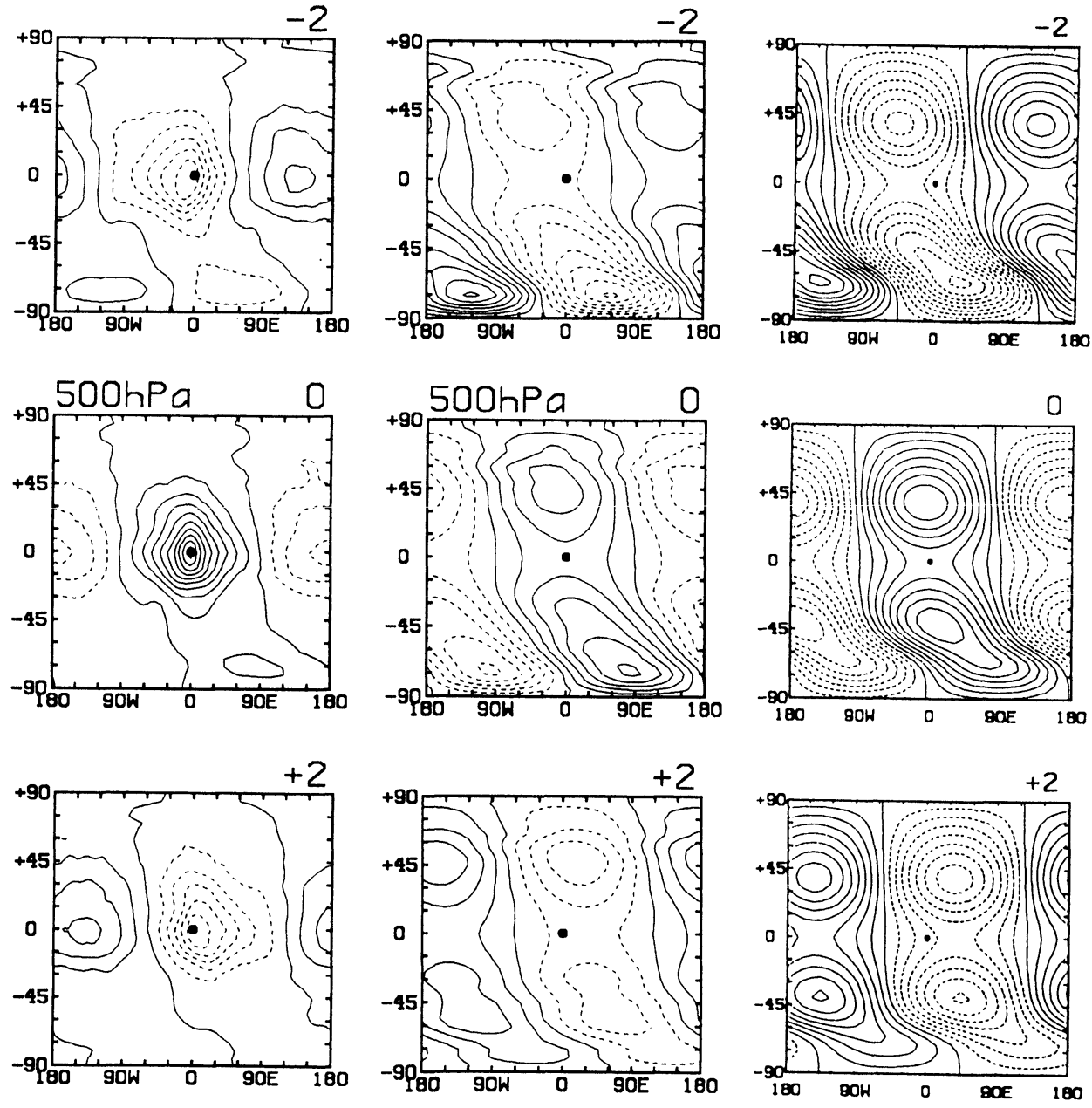

(a)

(b)

(c)

Fig. 1(a). One point lag-correlation maps at $500 \mathrm{hPa}$ level in $\mathrm{NH}$ summer. The abscissa and ordinate represent the longitude and latitude, respectively. Contour interval is $0.1 \mathrm{~m}$ and negative values are dashed. The numerals in upper right corners of each figure are days relative to the day of the middle figure. (b) Composite maps in the same level and season as in (a). (c) Composite maps of the wave pattern obtained in a numerical calculation of the Laplace's tidal equation with a standing-wave forcing in high latitudes of SH. 
istics of the 5-day wave. The correlation, however, decreases with latitudinal distance and finally vanishes at mid-latitudes. This result implies that the 5-day wave whose maximum amplitude should be located in the mid-latitudes is masked by large variability there.

Then, we investigated the detailed structure by means of a phase shift and compositing method. ${ }^{20)}$ Fig. 1(b) shows the composite maps in $500 \mathrm{hPa}$ levels in the $\mathrm{NH}$-summer. The reference latitude was chosen to be the Equator of $500 \mathrm{hPa}$ level. Note that the larger amplitude appears in the middle and high latitudes. The horizontal structures of the different levels differ little except for the slight increase of amplitude with height, indicating equivalent barotropic structure. From the lag-composite maps of 2 days before and after, we see that the overall pattern propagates westward with a period of nearly 5 days. Aside from the meridional NW-SE phase tilt and larger amplitude in the high latitudes of $\mathrm{SH}$, the horizontal wave pattern resembles the 5-day wave previously found in the atmosphere. ${ }^{2)}$ The average amplitude of the wave pattern at $45^{\circ} \mathrm{N}$ reaches $6-8$ geopotential meters, which is comparable to the results of others. ${ }^{2), 5), 6)} \mathrm{We}$ also performed the same analysis altering the reference latitude from the Equator to 45 degrees in both hemispheres to investigate the dependency of the wave pattern on the reference latitude. ${ }^{3), 21)}$ The results with reference latitudes lower than 30 degrees showed no significant difference, while those with reference latitudes higher than 30 degrees revealed a considerable modification in the wavestructure.

Numerical model. The free oscillation corresponding to the 5-day wave expressed by the Laplace's tidal equation does not contain the meridional phasetilt in SH observed in the atmosphere. On the other hand, it is not likely that there is a disturbance with the zonal wavenumber 1 and the period of 5 days which has no relation to the 5-day wave. In other words, the meridional phase tilt is considered to be related to the forcing mechanism of the 5-day wave. To confirm this idea, we investigate response characteristics of the barotropic atmosphere for various kinds of forcing.

We use the shallow water equations on a sphere (the Laplace's tidal equation) with a zonal flow $\bar{u}$ and the associated geopotential height field $\bar{\phi}$ :

$$
\begin{aligned}
& \frac{d U}{d t}=(f+\bar{\zeta}) V-\frac{g}{a} \frac{\partial \phi}{\partial \lambda}+F_{U}, \\
& \frac{d V}{d t}=-2 \bar{U} U \frac{u}{a\left(1-\mu^{2}\right)}-f U
\end{aligned}
$$

$$
\begin{gathered}
-\frac{g\left(1-\mu^{2}\right)}{a} \frac{\partial \phi}{\partial \mu}+F_{V}, \\
\frac{d \phi}{d t}=-\frac{V}{a} \frac{d \bar{\phi}}{d \mu}-(h+\bar{\phi}) D+F_{\phi}+Q,
\end{gathered}
$$

where

$$
\begin{aligned}
(U, V) & =(u, v) \cos \theta \\
\frac{d}{d t} & =\frac{\partial}{\partial t}+\frac{\bar{U}}{a\left(1-\mu^{2}\right)} \frac{\partial}{\partial \lambda} \\
D & =\frac{1}{a\left(1-\mu^{2}\right)}\left\{\frac{\partial U}{\partial \lambda}+\left(1-\mu^{2}\right) \frac{\partial V}{\partial \mu}\right\} \\
\bar{\zeta} & =-\frac{1}{a} \frac{d \bar{U}}{d \mu} .
\end{aligned}
$$

In these equations, $\mu=\sin \theta$ and $u$ and $v$ are zonal and meridional velocities, $\phi$ the deviation of free surface from the basic state, $\lambda$ longitude, $\theta$ latitude, $a$ radius of the Earth, $h$ mean fluid depth i.e. equivalent depth, $f$ the Coriolis parameter ( $=2 \Omega \sin \theta, \Omega$ being the rotation rate of the Earth), $g$ the gravitational acceleration, $F$ dissipation and $Q$ forcing, respectively. The equivalent depth is fixed as $10 \mathrm{~km}$, which corresponds to the isothermal atmosphere of $244 \mathrm{~K}$.

The forcing function is a standing wave given as

$$
Q=C \frac{\cos \theta}{\cos \theta_{0}} \exp \left\{-\left(\frac{\theta-\theta_{0}}{\Delta \theta}\right)^{2}\right\} G(\lambda, t) .
$$

The amplitude $C$ is given as $10 \mathrm{~m}$, which was determined by considering the observed amplitude of 5 -day waves in the high latitude of SH. The longitudinal and time functions are

$$
\begin{gathered}
G(\lambda, t)=\cos \left(\lambda-\lambda_{0}\right) A(t) \\
A(t)=\sum_{n=-6}^{n=14} \sin \left\{\left(\omega_{s}+n \delta \omega\right) t+\alpha_{n}\right\}
\end{gathered}
$$

and $\lambda_{0}=\pi / 16, \omega_{s}=2 \pi / 5$ days $^{-1}, \delta \omega=2 \pi / 100$ days $^{-1}$ and $\alpha_{\mathrm{n}}$ is the initial phase of each forcing. The period included in the band frequency forcing ranges approximately from 3 to 7 days. For simplicity, the Rayleigh friction is introduced as the dissipation, whose $e$ folding time is taken as a parameter. The time integration of the equations [1]-[3] were performed by means of the spectral method with associated Legendre polynomials as the orthogonal basis.

We specify a zonal flow $\bar{U}$. Then, the geopotential height field associated with this zonal flow is obtained 


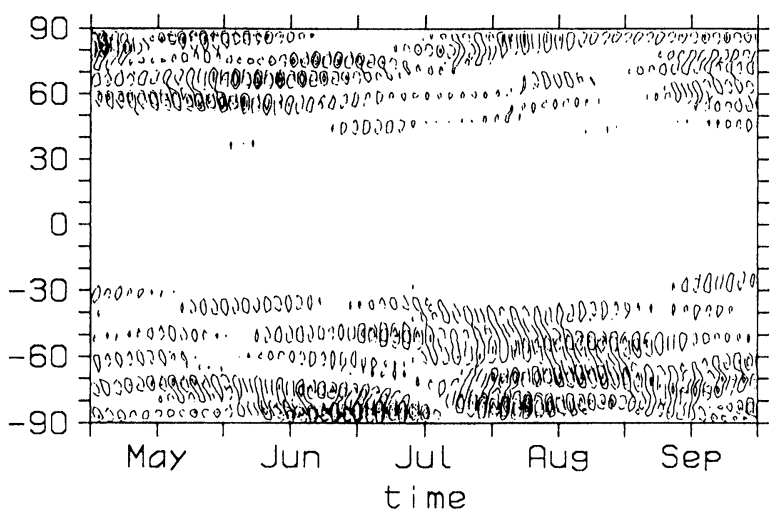

Fig. 2. Latitude-time diagram of the zonal flow which is oscillating with periods from 4.5 days to 5.6 days, in summer of 1988. The abscissa and ordinate denote the time and the latitude, respectively. Contour interval is $0.5 \mathrm{~m} / \mathrm{s}$.

by the geostrophic relation;

$$
\begin{aligned}
-\left(1-\mu^{2}\right) \frac{d \bar{\phi}}{d \mu} & =\frac{1}{\mathrm{~g}}\left\{\bar{U}^{2} \frac{\mu}{1-\mu^{2}}+a f \bar{U}\right\} \\
& \equiv K(\mu) .
\end{aligned}
$$

The zonal flow used in this study is similar to that shown in Fig. 3(b), which qualitatively resembles that at $500 \mathrm{hPa}$ level. The crudeness of the velocity profile may not be important, because the 5-day wave is not affected significantly by the zonal flow. This model contains 3 external parameters; the meridional location of the forcing $\theta_{0}$, the width of the forcing $\Delta \theta$ and the $e$-folding time scale of Rayleigh friction $v$. The standard parameters are taken as $\theta_{0}=65^{\circ} \mathrm{S}, \Delta \theta=10^{\circ}$ and $v=1 / 10$ days $^{-1}$. These values were used in the calculation if not stated otherwise.

The calculation showed an exponential growth in the first one period, followed by rather slow, but steady increase and eventually attains to an almost steady oscillation after 15 days. The characteristic horizontal pattern of the excited 5-day wave during one period is shown in Fig. 1(c). The numeral on the right upper corner of each map indicates days relative to the day of the middle figure. Note the large meridional phase tilt in $\mathrm{SH}$ whose pattern is similar to that in Fig. 1(b).

This hypothetical calculation mentioned above indicated that the standing oscillation of the atmosphere with the zonal wavenumber 1 and the period of 5 days located at high latitudes of SH produces a 5-day wave with the meridional phase tilt as seen in the observational results. Then, is there any real forcing mechanism that excites the standing oscillation in the high latitudes of SH?

The most probable candidate for this may be the interaction between a fluctuating zonal flow and the topography of zonal wavenumber 1 . Fig. 2 shows the latitude-time diagram of the zonal flow which is oscillating with periods of 4.5-5.6 days during SHsummer (April 16-October 15) of 1988. Note that the amplitude is largest in the high latitudes of both hemispheres. The amplitude is modulated with a time scale of about one month. On the other hand, the largest amplitude of the topography of zonal wavenumber 1 on the Earth is provided by the Antarctica as shown in Fig. 3(a). The mean zonal flow (solid line)

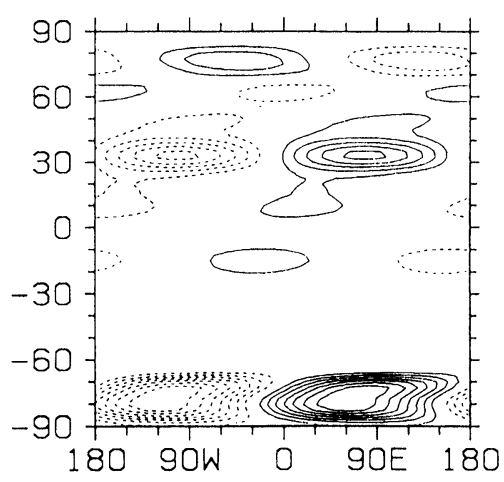

(a)

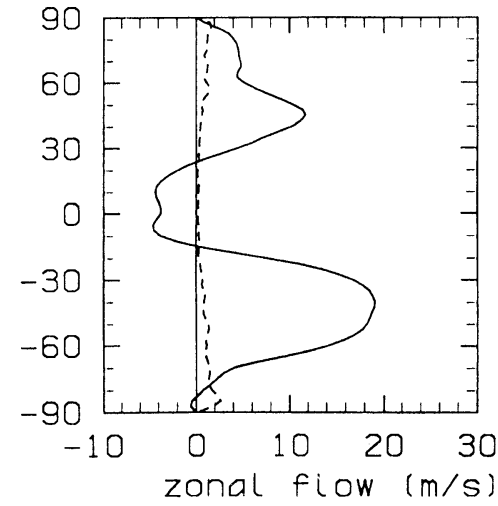

(b)

Fig. 3(a). The zonal wavenumber 1 component of the Earth's topography with contour interval of 200 meters. Negative values are shown with dashed lines. (b) The solid line represents the latitudinal variation of the averaged zonal flow in the same period of Fig. 2. The dashed line is the mean amplitude of the oscillating zonal flow with periods 4.5-5.6 days. 
and the mean amplitude of the zonal flow fluctuation with periods of 4.5-5.6 days (dashed line) during the same period of Fig. 2 are presented in Fig. 3(b).

Based on these observational facts we try to introduce a fluctuating zonal flow given as

$$
\bar{U}(\theta, t)=\bar{U}^{0}(\theta)+\bar{U}^{*}(\theta) \sin \omega_{s} t,
$$

in our model, where the subscript ${ }^{0}$ and $*$ denote the time average and the mean amplitude and $\omega_{s}=2 \pi / 5$ days $^{-1}$. We use the distribution shown in Fig. 3(b) in the calculation. It is unrealistic to introduce a zonal flow which oscillates synchronized in all latitudes as given in Eq. [12]. The latitudinal extent of the in-phase oscillation is at most 20 degrees in the real atmosphere as seen in Fig. 2. This unrealistic feature of the zonal flow, however, does not matter in our problem, because the maximum coupling of the topography and the zonal flow takes place only near the Antarctica. The mean zonal height field is calculated at every instant by the geostrophic relation stated above. Following Grose and Hoskins (1979), ${ }^{22)}$ we express the forcing term (Eq. [8]) as

$$
Q=\frac{\bar{U}}{a\left(1-\mu^{2}\right)} \frac{\partial \phi_{*}}{\partial \lambda},
$$

where $\phi_{*}$ is the zonal wavenumber 1 component of the Earth's topograph. The time integration was carried out for 30 days, because the fluctuation of the zonal flow with the period near 5 days is modulated by the order of one month period in Fig. 2. The average wave pattern (composite map) during last one period of the calculation is compared with the composite map of weather charts at $500 \mathrm{hPa}$ level in Fig. 4 in which (a) and (b) show the observational and theoretical results, respectively. Note that this model reproduced well the basic structure of the 5-day wave seen in the composite map of the weather charts. The amplitude of the wave excited in the model is also similar to that of the observational results, indicating that choice of the damping factor in the model is not bad. When we consider the simplicity of the model, the similarity of wave patterns is striking. This result suggests that the Laplace's tidal equation with a forcing at high latitudes of SH expresses quite well the structure of the 5-day wave.

Discussion. The 5-day wave is always generated in the atmosphere, but it cannot be seen due to background noises caused by fluctuation of the atmospheric circulation. In the first step of this study, we found that a signal of the 5 day wave is detectable in the tropics by the one-point correlation method. Although the amplitude of the 5-day wave is small in the tropics, the atmospheric noises are also small enough to detect the phase of the 5-day wave. The tropics is as if a window through which we can see a "tail" of the 5-day wave. Once we catch a tail (phase) of the 5-day wave, it is possible to visualize the global structure by superposing many "tails". Then, the image of the invisible main body emerges due to cancellation of noises.

By analyzing long-term data of geopotential height field according to this principle, we could visualize not only the structure of the 5-day waves, but also a characteristic structure related to the forcing mechanism, i.e., a NW-SE phase tilt which is large in $\mathrm{SH}$ and small in NH. The amplitude distribution in $\mathrm{NH}$ is very similar to the pure normal mode predicted by
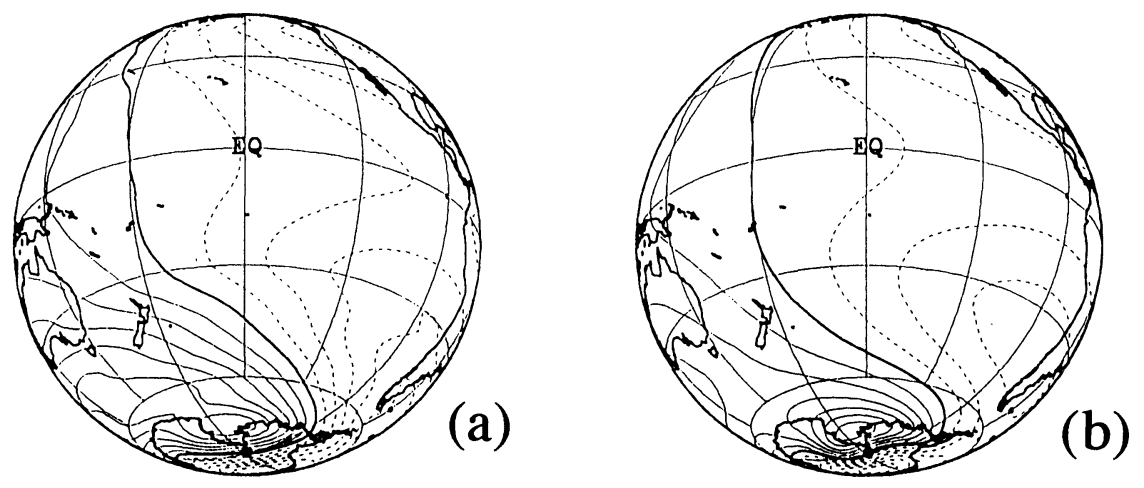

Fig. 4. Comparison of composited 5-day waves (a) The observation at $500 \mathrm{hPa}$ level in NH summer. (b) Numerical simulation incorporating the interaction between the fluctuating zonal flow and topography of zonal wavenumber 1. The longitude and latitude circles are drawn at every 30 degrees. Contour interval is 2 geopotential meters and negative values are shown with dashed lines. Solid circle denotes the South Pole. 
the theory. Lag-composite maps showed that the large amplitude first appears in the high latitude of $\mathrm{SH}$ and propagates into $\mathrm{NH}$. This implies the energy associated with the 5-day waves is emitted from the high latitude of SH. Then, we performed numerical experiments with shallow water equations on a sphere to confirm that the observed pattern of the 5-day waves is produced only when a periodic forcing is confined to the high latitudes.

We proposed an idea that such a forcing is produced by the interaction between the oscillating zonal flow and the topography of the Antarctica. Numerical simulations which incorporate this forcing mechanism reproduced a realistic 5-day wave with NW-SE phase tilt with reasonable amplitude.

The 5-day wave may be excited by any form of forcing, if it has the resonant frequency and the zonal wavenumber 1 . Therefore, there may be various forcing mechanisms. However, the 5-day waves excited by the random transient heat source in the tropics showed a considerable vertical phase tilt, ${ }^{16)}$ which is not consistent with observational facts. The source in the low and middle latitudes does not give rise to the meridional phase tilt. ${ }^{11)}$ On the other hand, the topographic effect of the Antarctica explains well both the vertical phase tilt and the meridional phase tilt in SH in addition to the main features of the free wave.

\section{References}

1) Longuet-Higgins, M. S. (1968) Phil. Trans. Roy. Soc. London, Ser. A 262, 511-607.

2) Madden, R., and Julian, P. (1972) JAS 29, 1464-1469.

3) Madden, R., and Julian, P. (1973) JAS 30, 935-940.

4) Madden, R., and Stokes, J. (1975) JAS 32, 831-836.

5) Madden, R. (1978) JAS 35, 1605-1618.

6) Ahlquist, J. (1982) JAS 39, 193-202.

7) Ahlquist, J. (1985) JAS 42, 2059-2068; Bourke, W. (1972) MWR 100, 683-689.

8) Lindzen, R., Straus, R., and Katz, B. (1984) JAS 41, 1320-1335.

9) Rodgers, C. D. (1976) JAS 33, 710-711.

10) Hirota, I., and Hirooka, T. (1984) JAS 41, 1253-1267.

11) Geisler, J., and Dickinson, R. E. (1976) JAS 33, 632-641.

12) Salby, M. (1981a) JAS 38, 1803-1826.

13) Salby, M. (1981b) JAS 38, 1827-1840.

14) Kasahara, A. (1980) JAS 37, 917-929.

15) Salby, M. (1984) Rev. Geophys. Space Phys. 22, 209-236.

16) Salby, M., and Garcia, R. (1987) JAS 44, 458-498.

17) Garcia, R., and Salby, M. (1987) JAS 44, 499-530.

18) Hamming, R. W. (1977) Digital filter. Eaglewood Cliffs, N. J. Prentis Hall Inc. p. 230.

19) Randel, W. J. (1988) TELLUS 40, 257-271.

20) Cai, M., and Van Den Dool, H. M. (1991) JAS 48, 1420-1436.

21) Deland, R. (1973) JAS 30, 934-940.

22) Grose, W. L., and Hoskins, B. J. (1979) JAS 36, 223-234. 